\title{
Utilidad y facilidad de uso percibida: desafíos tecnológicos en una modalidad b-learning
}

\author{
Utility and perceived ease of use: technological challenges of a b-learning modality
}

Norma Esther López Maldonado

Juan Carlos Valdés Godínes

\begin{abstract}
RESUMEN
Ante la cada vez más creciente demanda de matrícula en la educación superior en México, las instituciones educativas se han dado a la tarea de buscar opciones que permitan optimizar espacios y aprovechar los recursos existentes; el uso de un sistema de gestión de aprendizaje para complementar el proceso enseñanza y aprendizaje es una herramienta tecnológica muy valiosa para este fin. Tener éxito en la implementación de una modalidad de estudios apoyada con tecnología implica grandes desafíos, tales como que la tecnología propuesta sea aceptada y que los usuarios encuentren facilidad de uso en la misma. Este documento muestra los resultados de una investigación que tuvo como objetivo determinar la aceptación tecnológica y facilidad de uso percibida de un sistema de gestión del aprendizaje para una modalidad b-learning, utilizado como prueba piloto en estudiantes del preuniversitario de la Facultad de Contaduría y Administración C-I en la Universidad Autónoma de Chiapas, en México. Para ello se utilizó el enfoque mixto no experimental, con diseño transversal tipo exploratorio; para la elaboración del instrumento se utilizó el modelo de aceptación tecnológica. Los resultados muestran una tendencia positiva a la aceptación de la tecnología propuesta, pero con áreas de mejora en cuanto a la utilidad percibida.
\end{abstract}

Palabras clave: educación superior, diseño instruccional, proceso enseñanza y aprendizaje, sistemas de gestión del aprendizaje, tecnologías de la información y de la comunicación.

\section{ABSTRACT}

Faced with the ever increasing demand for enrollment in Higher Education in Mexico, educational institutions have set out to find options that allow optimizing spaces and taking advantage of existing resources; the use of a learning management system to complement the teaching-learning process is a very valuable technological tool for this purpose. Being successful in implementing a technology-supported study modality implies great challenges, among them that the proposed technology is accepted, and that users find ease of use in it. This document shows the results of an investigation aimed to determine the technological acceptance and perceived ease of use of a learning management system for a b-learning modality, used as a pilot test in pre-university students of the Facultad de Contaduría y Administración C-I at the Universidad Autónoma de Chiapas, in Mexico. For this, the non-experimental mixed approach was used, with an exploratory-type cross-sectional design; the Technological Acceptance Model was used to elaborate the instrument. The results show a positive trend towards acceptance of the proposed technology, but with areas of improvement in terms of perceived utility.

Keywords: higher education, instructional design, teachinglearning process, learning management systems, information and communication technologies. 


\section{INTRODUCCIÓN}

La globalización, producto de los avances científicos y tecnológicos de la sociedad actual, invita a la reorganización de las actividades y quehaceres aprovechando el uso de las tecnologías de información y comunicación (TIC). En este sentido el ámbito educativo no es la excepción, por lo que resulta importante reconocer la trascendencia que puede tener la utilización de las herramientas tecnológicas en los ambientes de enseñanza y de aprendizaje, ya que su uso colabora con el desarrollo de competencias en los estudiantes a la vez que contribuye con la desaparición de barreras relativas a tiempo y espacio.

Con base en lo mencionado, surge la necesidad de construir nuevos escenarios que coadyuven a mejorar el proceso de enseñanza y aprendizaje (PEA) utilizando TIC, pero concebidos como sistemas abiertos que permitan la construcción del conocimiento de manera sistémica e integrada, que consideren las necesidades de los estudiantes, de tal forma que les permitan tener un rol más activo, con actitud crítica y proactiva, incrementen su curiosidad y la libre discusión de ideas.

En este sentido, los sistemas de gestión de aprendizaje (Learning Management Systems, mejor conocidos como LMS) han permitido implementar nuevas modalidades de estudio que permiten aprovechar las bondades del uso de la tecnología y del internet.

Los LMS son vistos tanto como una puerta de salida para solventar las necesidades institucionales (en cuanto a la ampliación de la matrícula) como también una oportunidad para la creación de nuevos escenarios que permiten la interacción de los estudiantes, potenciando de esta manera sus capacidades y habilidades natas, así como las futuras.

Uno de los segmentos de mayor aceptación y crecimiento en la educación superior, en cuanto al uso tecnología, es la combinación de clases presenciales con apoyo de tecnología basada en la web (Belanger y Jordan, 2000); en este sentido, y según Graham (2013), la convergencia entre el entorno de aprendizaje presencial y el entorno virtual permite ampliar y modificar las posibilidades de comunicación e interacción. Estos

Norma Esther López Maldonado. Directora de Formación e Investigación Educativa y profesora de asignatura del Centro de Estudios para el Desarrollo Municipal y Políticas Públicas de la Universidad Autónoma de Chiapas (México). Es candidata a doctora en Innovación en Tecnología Educativa; maestra en Tecnología Educativa, y maestra en Administración por el ITESM. Diplomado Evaluación de la calidad de programas y cursos a distancia. Diplomado internacional: La evaluación de la calidad de programas de Educación a Distancia. Correo electrónico: norma.lopez@unach.mx. ID: https://orcid.org/0000-0003-3726-4926.

Juan Carlos Valdés Godínes. Profesor-investigador del Centro Interdisciplinario de Investigación y Docencia en Educación Técnica, Querétaro, México. Es doctor en Pedagogía por la UNAM y docente de los doctorados en Tecnología Educativa e Innovación y Tecnología Educativa, y de la maestría en Didáctica de las Matemáticas y de las Ciencias de la Universidad Autónoma de Querétaro. Cuenta con reconocimiento al perfil deseable ProdeP; responsable del Cuerpo Académico en Consolidación "Estudios en Cultura Digital, sus Implicaciones en la Educación y Difusión de la Ciencia”. Correo electrónico: jvaldesgodines@ gmail.com. ID: https://orcid.org/ 0000-0003-4488-7638. 
sistemas educativos, en los que se combinan las clases presenciales con la instrucción mediada por tecnología, son conocidos como b-learning (Graham, 2006).

$\mathrm{Al}$ respecto, Gisbert, De Benito, Pérez y Salinas (2018) mencionan que el concepto b-learning ha tenido diferentes denominaciones (híbrido, semipresencial, mixto y mezclado) y diferentes visiones a lo largo del tiempo, entre las que puede ser considerado como (p. 196):

a) Combinación de aprendizaje presencial-online.

b) Combinación de sistemas de distribución o tecnologías de distribución de formación.

c) Combinación de estrategias o modelos de aprendizaje.

Adicionalmente, el b-learning también puede ser clasificado en modelos basados en la combinación de modalidades de distribución, de combinación de métodos didácticos o la combinación de instrucción cara-a-cara con instrucción en línea; Salinas, Darder y De Benito (2015) los clasifican en dos: de rotación y flexible. Cada uno de estos modelos presenta ciertas características que lo definen. En el de rotación los estudiantes pueden rotar entre modalidades de aprendizaje, entre clase o sitio, entre laboratorio, en aula invertida, o en rotación individual (de acuerdo a la organización del profesor); mientras que el flexible se caracteriza por que el aprendizaje es el centro del proceso formativo, y los estudiantes pueden cambiar de modalidad de acuerdo a sus intereses, generando otros modelos, como por ejemplo: flexible, a la carta o virtual enriquecido (Salinas, Darder y De Benito, 2015).

Estos modelos mencionados, según Adell y Area (2009), se utilizan en las instituciones de educación superior, y permiten brindar flexibilidad en tiempo y espacio, fomentar la interacción entre estudiante y docente, motivar la autonomía y sentido de responsabilidad respecto a su proceso de aprendizaje, además de fomentar el desarrollo de las competencias digitales; por otra parte, para las instituciones de educación superior el uso del b-learning coadyuva a la mejora educativa y eficiencia económica, permite mejorar la educación, incrementa el acceso y la flexibilidad, y la relación costo-beneficio (Graham, 2013). Si bien los beneficios en cuanto al b-learning son muchos, para las instituciones de educación superior se perciben dos muy importante, uno enfocado a la parte económica, específicamente lo referente a la reducción de costos, y el otro relativo a la mejora del proceso enseñanza y aprendizaje, y que en su conjunto resultan complementarios.

En este sentido, resulta necesario tomar en consideración los elementos que conforman a los ambientes de aprendizaje, mismos que están constituidos por dos tipos, los denominados constitutivos y los llamados conceptuales; cuando se habla de elementos constitutivos se hace referencia a medios de interacción, recursos, factores ambientales y factores psicológicos; mientras que los conceptuales se refieren netamente a aquellos aspectos que tienen que ver con el concepto de ambiente virtual en sí, compuesto por el diseño instruccional y la interfaz (Herrera, 2006). 
En este punto centraremos nuestra atención en el diseño instruccional, que según Williams, Schrum, Sangrà y Guàrdia (2003) es un proceso que permite planificar tanto los materiales educativos como la secuencia y dosificación de estos, de tal forma que ayuden al logro del aprendizaje en el estudiante; por ello se debe considerar la relación integral de todos los elementos en el material a desarrollar, para que el ambiente de aprendizaje sea óptimo.

El ambiente virtual estará determinado tanto por el diseño instruccional como por el diseño de la interfaz, este último está relacionado con el elemento visual que colaborará en la presentación de un ambiente agradable para el trabajo tanto del docente como del estudiante, en donde se debe definir el orden visual de cómo quedarán establecidos los elementos que contempla el ambiente de aprendizaje a elaborar.

En la actualidad podemos observar cómo las instituciones educativas del mundo entero utilizan cada vez más modelos pedagógicos del tipo b-learning, pero ello no siempre es sinónimo de éxito asegurado, de hecho también existe la deserción al igual que en los modelos presenciales. Las razones para que un estudiante abandone un entorno virtual de aprendizaje, según Vázquez y Rodríguez (2007), pueden ser variadas, como por ejemplo la falta de compromiso académico e identificación profesional por parte del estudiante o los factores socioeconómicos y demográficos, sin embargo muy pocas instituciones educativas se han dado a la tarea de verificar el grado de aceptación que tienen los estudiantes respecto a la tecnología que sirve de base para la implementación de la modalidad b-learning.

Por todo lo dicho anteriormente, la modalidad b-learning no consiste en trasladar el modelo presencial a la virtualidad sino que implica utilizar un modelo de diseño instruccional ad-hoc que permita generar un ambiente de trabajo en el cual los resultados se vean reflejados en la adquisición de competencias por parte de los estudiantes, lo cual se permee a través de la aceptación tecnológica de los estudiantes en el LMS utilizado.

\section{Interrogantes y objetivos}

Las interrogantes que dieron origen a la presente investigación son las siguientes:

- ¿Cuál es la utilidad y la facilidad de uso percibida por los estudiantes de un LMS utilizado para una modalidad b-learning?

- ¿Cuáles son los parámetros culturales y sociales que influyen en la percepción de utilidad y facilidad de uso de un LMS utilizado para una modalidad b-learning?

El objetivo general de este estudio es determinar la utilidad y facilidad de uso percibida de un LMS utilizado para una modalidad tipo b-learning en alumnos del curso preuniversitario en la Facultad de Contaduría y Administración Campus I, de la Universidad Autónoma de Chiapas. 
Como objetivos específicos se consideran los siguientes:

1. Evaluar la influencia social en cuanto a la utilidad y facilidad de uso percibida del LMS.

2. Evaluar la influencia cultural en cuanto a la utilidad y facilidad de uso percibida de un LMS.

\section{Metodología}

El presente documento muestra los resultados obtenidos de una investigación para determinar la aceptación y facilidad hacia el uso de un LMS, así como la incidencia de la influencia social y cultural, de los estudiantes del preuniversitario de una institución de educación superior en México, que participaron en una prueba piloto en modalidad tipo b-learning, utilizando el software de uso libre Moodle. El diseño instruccional utilizado para la planeación de contenidos, recursos didácticos y evaluación en la prueba piloto es el denominado ADDIE. El diseño recibe su nombre con base en las cinco etapas que lo componen y que consisten en el análisis, diseño, desarrollo, implementación y evaluación (Belloch, 2014). El modelo ADDIE es genérico, las etapas pueden realizarse de manera secuencial, ascendente o simultánea.

El instrumento para la recogida de datos fue realizado tomando como marco de referencia el modelo de aceptación tecnológica (TAM), adaptado de acuerdo a las necesidades requeridas, debido a que para predecir el grado de aceptación y la facilidad en cuanto al uso de una tecnología determinada existen modelos probados previamente, y uno de ellos es el mencionado TAM, propuesto por Davis (1989).

El TAM está basado en la Teoría de Acción Razonada (TRA) de Ajzen (1991) y Ajzen y Fishbein (1980), conocida también como modelo de comportamiento planeado, que sirve para predecir el comportamiento de las personas, con base en sus actitudes e intenciones. Este modelo asume que cuando los usuarios se enfrentan a una nueva tecnología existen varios factores que pueden influir en el grado de aceptación, como la utilidad percibida, la facilidad de uso y el disfrute percibidos.

Davis (1989) menciona que la utilidad percibida y la facilidad de uso percibida representan las convicciones que llevan a aceptar a la tecnología como parte de un modelo. Por su parte, Martín y Sánchez (2014) mencionan que el efecto positivo de las variables antes mencionadas sobre la adopción de variadas innovaciones tecnológicas considerando el contexto del e-learning y b-learning ha sido probado empíricamente en diversas ocasiones, lo que ha implicado la realización de modificaciones al modelo de aceptación tecnológica, con la intención de extender y valorar la incorporación de otras variables al modelo; entre esas variaciones se incluyen la influencia social y la influencia cultural, por lo que, para comprender la importancia de las variables que se utilizan en la realización de la presente investigación, a continuación se describen 
brevemente las principales modificaciones que ha sufrido dicho modelo a lo largo del tiempo:

- Una de las modificaciones da como resultado el llamado TAM2 (Venkatesh y Davis, 2000), en el cual se explica la utilidad percibida y la intención hacia el uso, pero en términos de la influencia social y de los procesos cognitivos, agregando las variables: norma subjetiva, voluntariedad, imagen, experiencia, relevancia en el trabajo, calidad de la salida y demostrabilidad de resultados.

- El TAM3, por su parte, representa una extensión del TAM2, y menciona que la facilidad de uso percibida está determinada por las variables: ansiedad frente a la tecnología, gozo frente a la tecnología, percepción de disfrute, usabilidad objetiva y percepción de control externo. Venkatesh y Bala (2008) establecen una conexión del TAM3 con la perspectiva de los modelos de difusión de la innovación (IDT), tomando en consideración aspectos cognitivos individuales fundamentados en el TAM, los atributos propios de las organizaciones, las características de los sistemas organizativos y los aspectos contextuales o situacionales, dando apertura a la valoración y peso de la incorporación de la personalidad y características sociodemográficas, creencias internas, la experiencia previa, hábitos y rutinas de los sujetos analizados, los cuales pueden intervenir en el comportamiento hacia una innovación determinada; adicional a ello se evalúan factores externos como la influencia del sistema y alguna otra variable que no se consideraba inicialmente en este modelo. Además, Malhotra y Galleta (1999) proponen una extensión para considerar la influencia social.

- Por su parte, Zakour (2004) y McCoy y Polak (2003) proponen una extensión que tome en consideración la influencia cultural, de la cual pretenden entender cómo las diferencias sociales y culturales pueden predecir el comportamiento frente a una tecnología determinada, considerando siete procesos culturales.

La técnica utilizada para recabar información fue la encuesta, misma que estuvo compuesta por 27 ítems, elaborados con base en el modelo de aceptación tecnológica extendido para considerar la influencia social y cultural, a partir de cuatro variables que se presentan en la tabla 1 , en donde también se señala el número de ítems que se abordan por cada variable.

Tabla 1. Ítems por variable.

\begin{tabular}{|c|c|}
\hline Variable & Número de ítems \\
\hline 1. Utilidad percibida & 6 \\
\hline 2. Facilidad de uso percibida & 6 \\
\hline 3. Influencia social & 3 \\
\hline 4. Influencia cultural & 7 \\
\hline Total & 22 \\
\hline
\end{tabular}

Fuente: Construcción propia. 
La variable "Utilidad percibida" pretende medir el grado en que una persona considera que utilizando un sistema determinado mejorará su desempeño en el mismo, mientras que la "Facilidad de uso percibida" se refiere al grado en el que una persona considera que utilizando un sistema determinado realizará menos esfuerzo para el desarrollo de sus actividades en el mismo (Yong, Rivas y Chaparro, 2010).

Por su parte, la variable llamada "Influencia social", vista desde los procesos que se abordan, tiene un efecto directo en la actitud de los usuarios y efectos indirectos en la intención hacia el uso a través de la actitud (Leyton, 2013), mientras que la variable "Influencia cultural" trata de explicar el mecanismo mediante el que las diferencias culturales puedan predecir el comportamiento frente a una tecnología determinada (Leyton, 2013).

Esta encuesta se estructuró en dos secciones, en la primera se recupera información relativa al género, lugar de residencia, lugar de nacimiento y nivel socioeconómico percibido, con la intención de determinar la relación entre estas variables y la aceptación de los estudiantes en torno a la tecnología propuesta; en la segunda sección se recupera información relativa a las cuatro variables, a saber: utilidad percibida, facilidad de uso percibida, influencia social, influencia cultural.

Para determinar la confiabilidad de la encuesta se calculó el coeficiente alfa de Cronbach en una muestra aplicada a 24 estudiantes. De acuerdo a la teoría clásica, la confiabilidad consiste en determinar el grado en que un instrumento que contiene varios ítems puede medir de manera consistente una muestra de la población, es decir, "el grado en que una medida está libre de errores" (Celina y Campo-Arias, 2005, p. 574).

El coeficiente alfa de Cronbach se utiliza para medir la correlación que existe entre los ítems que componen un instrumento, con la ventaja de que se requiere de una sola aplicación del instrumento para probar su confiabilidad, basado en la medición de la respuesta del sujeto con respecto a los ítems del instrumento, y se estima con la siguiente fórmula:

$$
\alpha=\frac{K}{K-1}\left[1-\frac{\sum S_{i}{ }^{2}}{S_{T}{ }^{2}}\right]
$$

En donde:

$K=$ Número de ítems.

$S_{i}^{2}=$ Sumatoria de varianzas de los ítems.

$S_{T}{ }^{2}=$ Varianza de la suma de los ítems.

$\alpha=$ Coeficiente de alfa de Cronbach.

El coeficiente alfa de Cronbach oscila entre 0 y 1 . Si el resultado se encuentra próximo a 1 los ítems serán más consistentes entre sí. En esta ocasión se realizó el 
cálculo en forma manual y se obtuvo un coeficiente de 0.948 , lo cual permitió corroborar que el instrumento es confiable.

Aprovechando las bondades que brinda el uso de tecnología, tanto para el procesamiento de datos como para ratificar el coeficiente de Cronbach se utilizó el programa SPSS V.19 (Statistical Package for the Social Sciences). Este software es una herramienta ampliamente utilizada para el tratamiento y análisis de datos estadísticos, adaptado al entorno Windows, que permite visualizar la información a través de pantallas que contienen una serie de opciones para realizar de manera automatizada el cálculo de diferentes variables.

La encuesta se aplicó a un total de 88 estudiantes participantes en la prueba piloto, utilizando un formulario de Google, con respuestas en escala de Likert. Para el análisis de datos de esta investigación se obtuvieron dos medidas de tendencia central: la media aritmética y la moda, así como una de dispersión, la desviación estándar.

La media es única, y nos ayuda a determinar el centro de gravedad de toda la distribución de datos representados en los valores que se observan. La moda nos ayuda a observar el dato que más se repite o que ocurre con mayor frecuencia en todas las variables, mientras que la desviación estándar, como medida de dispersión central, nos sirve para medir la variabilidad de la dispersión de los datos alrededor de la media.

\section{Resultados}

De acuerdo con los resultados obtenidos, según se observa en la tabla 2, el 47.7\% de los encuestados son mujeres y el 52.3\% son hombres; el $77.3 \%$ cuenta con edades que oscilan entre 18 y 20 años, mientras que el 22.7\% entre 21 y 23 años. El 58\% mencionó que nació en un entorno urbano, en contraste con el $42 \%$ que afirma haber nacido en un entorno rural.

Tabla 2. Datos generales.

\begin{tabular}{|c|c|c|c|c|c|c|c|}
\hline \multicolumn{2}{|c|}{ Participantes } & \multicolumn{2}{|c|}{ Edad } & \multicolumn{2}{|c|}{ Lugar de residencia } & \multicolumn{2}{|c|}{ Lugar de nacimiento } \\
\hline Género & Total & $18-20$ & $21-24$ & Dentro de la ciudad & Fuera de la ciudad & Urbano & Rural \\
\hline Mujeres & 42 & 33 & 9 & 27 & 15 & 22 & 20 \\
\hline Hombres & 46 & 35 & 11 & 35 & 11 & 29 & 17 \\
\hline Total & 88 & 68 & 20 & 62 & 26 & 51 & 37 \\
\hline
\end{tabular}

Fuente: Construcción propia.

En cuanto al lugar de residencia, el 70.5\% afirmó vivir dentro de la ciudad, mientras que el resto fuera de ella; esto significa que independientemente del género y de que el $42 \%$ nació en un entorno rural, actualmente en su mayoría habitan en un entorno urbano; mientras que más del 56\% afirma ser de nivel socioeconómico 
medio y solo menos del 10\% afirma pertenecer a un nivel bajo, tal como se aprecia en la tabla 3.

Tabla 3. Nivel socioeconómico.

\begin{tabular}{|c|c|c|c|c|c|c|}
\hline \multicolumn{2}{|c|}{ Participantes } & \multicolumn{5}{|c|}{ Nivel socioeconómico } \\
\hline Género & Total & Bajo & Medio-bajo & Medio & Medio-alto & Alto \\
\hline Mujeres & 42 & 4 & 15 & 23 & 0 & 0 \\
\hline Hombres & 46 & 4 & 6 & 27 & 9 & 0 \\
\hline Total & 88 & 8 & 21 & 50 & 9 & 0 \\
\hline
\end{tabular}

Fuente: Construcción propia.

\section{Utilidad percibida}

Los resultados más sobresalientes respecto a la variable "Utilidad percibida" y que se aprecian en la figura 1 son que el 49\% de los estudiantes menciona que está totalmente de acuerdo en la importancia el trabajo del profesor en cuanto a promover en los estudiantes el uso de la plataforma, mientras que el 18\% está de acuerdo. El 24\% de los estudiantes está totalmente de acuerdo en cuanto a su entusiasmo por utilizar la plataforma, mientras que el 19\% está solo de acuerdo. El 34\% de los estudiantes está de acuerdo con que el trabajo en plataforma les ha permitido mejorar sus calificaciones, y solo el 9\% está totalmente de acuerdo. Además, el 15\% está totalmente de acuerdo, mientras que el $26 \%$ está de acuerdo en que el uso de plataforma les

\section{Variable Utilidad percibida}

$\begin{array}{llllllllllll}0 \% & 10 \% & 20 \% & 30 \% & 40 \% & 50 \% & 60 \% & 70 \% & 80 \% & 90 \% & 100 \%\end{array}$

Es importante que mi maestro me motive a utilizar la plataforma

Estoy entusiasmado(a) con la idea de utilizar la plataforma El uso de la plataforma me permite ser mejor estudiante

Mi experiencia usando plataformas educativas similares es amplia

La plataforma me permite cumplir de mejor manera con mi responsabilidad como estudiante

Con el uso de la plataforma he mejorado mis calificaciones

- Totalmente en desacuerdo En acuerdo
En desacuerdo

Figura 1. Resultados variable "Utilidad percibida"

Fuente. Construcción propia. 
permite ser mejor estudiante. En cuanto a la experiencia previa utilizando plataformas educativas similares, el 16\% manifestó estar totalmente de acuerdo y el 18\% dijo estar de acuerdo. Por otra parte, el 25\% estuvo totalmente de acuerdo, mientras que el 26\% estuvo de acuerdo en que la plataforma les permite cumplir mejor con su responsabilidad como estudiante; mientras que solo el 9\% estuvo totalmente de acuerdo y el $34 \%$ de acuerdo en que el uso de plataforma les ha permitido mejorar sus calificaciones.

Por otra parte, con la intención de ubicar e identificar el punto sobre el cual se centran y hacia a donde se inclinan o se agrupan más los datos analizados, en la tabla 4 se muestran los resultados obtenidos a partir de la media, la moda y la desviación estándar.

Para realizar este análisis se asignó a cada opción de respuesta una puntuación del 1 al 5, en donde 5 es totalmente de acuerdo, 4 de acuerdo, 3 ni de acuerdo ni en desacuerdo, 2 en desacuerdo y 1 totalmente en desacuerdo.

De acuerdo al análisis realizado se encontró que la variable "Utilidad percibida" presenta una media de 3.30 con una moda de 3 y una desviación estándar de 1.27; esto significa que de manera general las respuestas que más se repitieron estuvieron en la opción ni de acuerdo ni en desacuerdo, pero como la media se encuentra por encima del 3 (ni de acuerdo ni en desacuerdo), eso significa que existe utilidad hacia el uso de la tecnología propuesta.

Además, de los resultados de esta variable resaltan dos cosas: la primera es lo relativo al ítem de la importancia que le dan los estudiantes a que el maestro los motive a utilizar la plataforma, ya que presenta una moda de 5 (totalmente de acuerdo) con una media de 3.98, ligeramente por encima de la media total de esta variable, y con una desviación estándar de 1.22, la segunda menor de toda la variable; la segunda son los resultados relativos al ítem "con el uso de la plataforma he mejorado mis calificaciones", debido a que presenta una moda de 4 (de acuerdo) con una media de 3.13 (por debajo del resultado global de la variable completa) y una desviación estándar de 1.18, la menor de todos los ítems de esta variable, tal como se aprecia en la tabla 4.

Tabla 4. Media, desviación estándar y moda, de la variable "Utilidad percibida".

\begin{tabular}{|c|c|c|c|}
\hline Ítem & $\bar{x}$ & $\sigma$ & Moda \\
\hline 1. Es importante que mi maestro me motive a utilizar la plataforma & 3.98 & 1.22 & 5 \\
\hline 2. Estoy entusiasmado(a) con la idea de utilizar la plataforma & 3.24 & 1.36 & 3 \\
\hline 3. El uso de la plataforma me permite ser mejor estudiante & 3.07 & 1.31 & 3 \\
\hline 4. Mi experiencia usando plataformas educativas similares es amplia & 2.99 & 1.26 & 3 \\
\hline $\begin{array}{l}\text { 5. La plataforma me permite cumplir de mejor manera con mi } \\
\text { responsabilidad como estudiante }\end{array}$ & 3.43 & 1.28 & 3 \\
\hline 6. Con el uso de la plataforma he mejorado mis calificaciones & 3.13 & 1.18 & 4 \\
\hline
\end{tabular}




\section{Facilidad de uso percibida}

En la variable "Facilidad de uso percibida", tal como se observa en la figura 2, se encontró que solo el 9\% de los estudiantes estuvo totalmente de acuerdo en que su interacción con la plataforma les provoca ansiedad, seguido del 19\% que estuvo de acuerdo; mientras que el 14\% de los estudiantes manifestaron que les gusta realizar sus actividades académicas utilizando la plataforma, seguido por el $28 \%$ que dijo estar de acuerdo. Por otra parte, el $24 \%$ considera estar totalmente de acuerdo en que utilizar la plataforma les permite ser más eficientes en la entrega de sus tareas escolares, seguido del 28\% que afirmó estar de acuerdo. En cuanto al ítem que menciona el disfrute de utilizar la plataforma como apoyo a clases presenciales, el 18\% de los estudiantes manifestó estar totalmente de acuerdo, seguido del 33\% que mencionó estar de acuerdo. Con relación a la percepción acerca de que el uso de la plataforma les permite realizar de manera más fácil las tareas, el 17\% dijo estar totalmente de acuerdo, mientras que el 30\% mencionó estar de acuerdo. Por otra parte, el 27\% manifestó estar totalmente de acuerdo en que la plataforma cuenta con un equipo de profesionales que respaldan tanto la parte tecnológica como la académica, seguido del 25\% que dijo estar de acuerdo.

\section{Variable Facilidad de uso percibida}

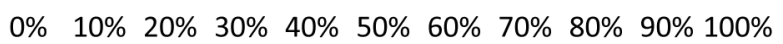

Mi interacción con la plataforma me provoca ansiedad

Me gusta realizar mis actividades académicas utilizando la plataforma

Considero que utilizar la plataforma me permite ser más eficiente en la entrega de mis tareas Disfruto utilizar la plataforma como apoyo a mis clases presenciales

Utilizar la plataforma me permite realizar mis tareas de manera más fácil

La plataforma cuenta con un equipo de profesionales que respaldan tanto la parte tecnológica como la académica

- Totalmente en desacuerdo

En desacuerdo

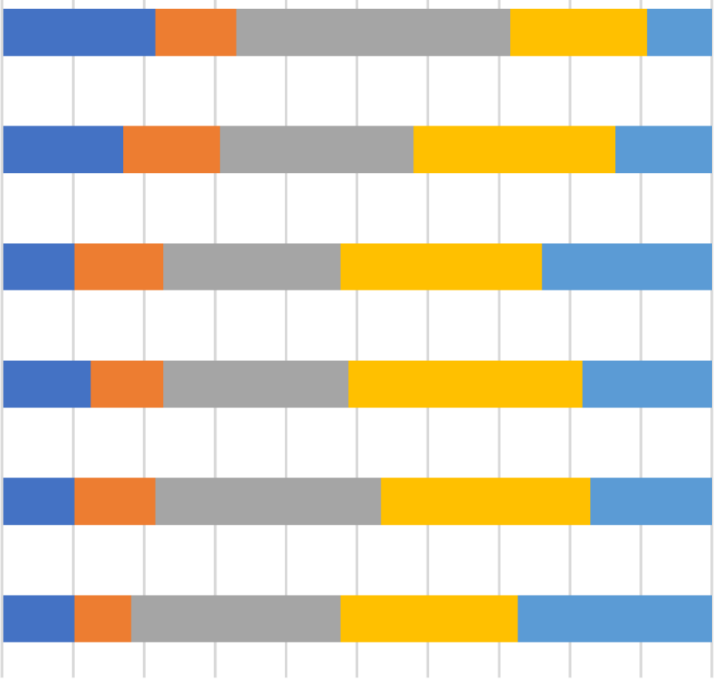

En acuerdo

Ni de acuerdo ni en desacuerdo

- Totalmente de acuerdo

Figura 2. Resultados variable "Facilidad de uso percibida".

Fuente: Construcción propia. 
Para concluir el análisis de esta variable, de igual forma que con la anterior, se obtuvieron la media, desviación estándar y moda; de manera global, la media fue de 3.25 , con una desviación estándar de 1.25 , y para esta variable el resultado de la moda fue tipo bimodal en 3 y 4, como se observa en la tabla 5. La mayor dispersión de datos se observó en el ítem relativo a "Me gusta realizar mis actividades académicas utilizando la plataforma”, con 1.29 de desviación estándar, un poco por arriba del promedio general de esta variable que fue de 1.25; mientras que la menor distribución de datos se observó en el ítem "Disfruto utilizar la plataforma como apoyo a mis clases presenciales", con una desviación estándar de 1.19.

Tabla 5. Media, desviación estándar y moda, variable "Facilidad de uso percibida".

\begin{tabular}{|c|c|c|c|}
\hline Ítem & $\bar{x}$ & $\sigma$ & Moda \\
\hline 1. Mi interacción con la plataforma me provoca ansiedad & 2.83 & 1.23 & 3.00 \\
\hline 2. Me gusta realizar mis actividades académicas utilizando la plataforma & 3.08 & 1.29 & 4.00 \\
\hline $\begin{array}{l}\text { 3. Considero que utilizar la plataforma me permite ser más eficiente } \\
\text { en la entrega de mis trabajos }\end{array}$ & 3.43 & 1.27 & 4.00 \\
\hline 4. Disfruto utilizar la plataforma como apoyo a mis clases presenciales & 3.34 & 1.25 & 4.00 \\
\hline 5. Disfruto utilizar la plataforma como apoyo a mis clases presenciales & 3.32 & 1.19 & 3.00 \\
\hline 6. Utilizar la plataforma me permite realizar mis tareas de manera más fácil & 3.51 & 1.26 & 3.00 \\
\hline
\end{tabular}

$$
\text { Fuente: Construcción propia. }
$$

Del análisis total de esta variable se deduce que se percibe facilidad de uso, ya que la media se ubica por encima de 3, lo que implica que la mayoría de los estudiantes está de acuerdo.

\section{Influencia social}

Referente a los resultados de la variable "Influencia social", tal como se observa en la figura 3, solo el 9\% de los estudiantes manifestó estar totalmente de acuerdo en que utilizar la plataforma les permite obtener mejores calificaciones, y el 30\% manifestó estar de acuerdo. El 8\% manifestó estar totalmente de acuerdo en que el uso de la plataforma les ayuda a relacionarse con sus compañeros de clase, mientras que el 18\% seleccionó de acuerdo. Por otra parte, solo el 8\% manifestó estar totalmente de acuerdo en que el uso de plataforma cumple con sus expectativas como estudiantes, seguido del 33\% que manifestó estar de acuerdo.

Respecto a la media de esta variable, se ubicó en 2.96, con una desviación estándar promedio de 1.16 y una moda de 3 , lo que significa que existe indiferencia en cuanto a que exista influencia social en el uso de la plataforma, pues el porcentaje mayor de respuestas de todas las variables está en la opción de respuesta ni de acuerdo ni en desacuerdo, tal como se observa en la tabla 6. Algo que llama la atención de estos 


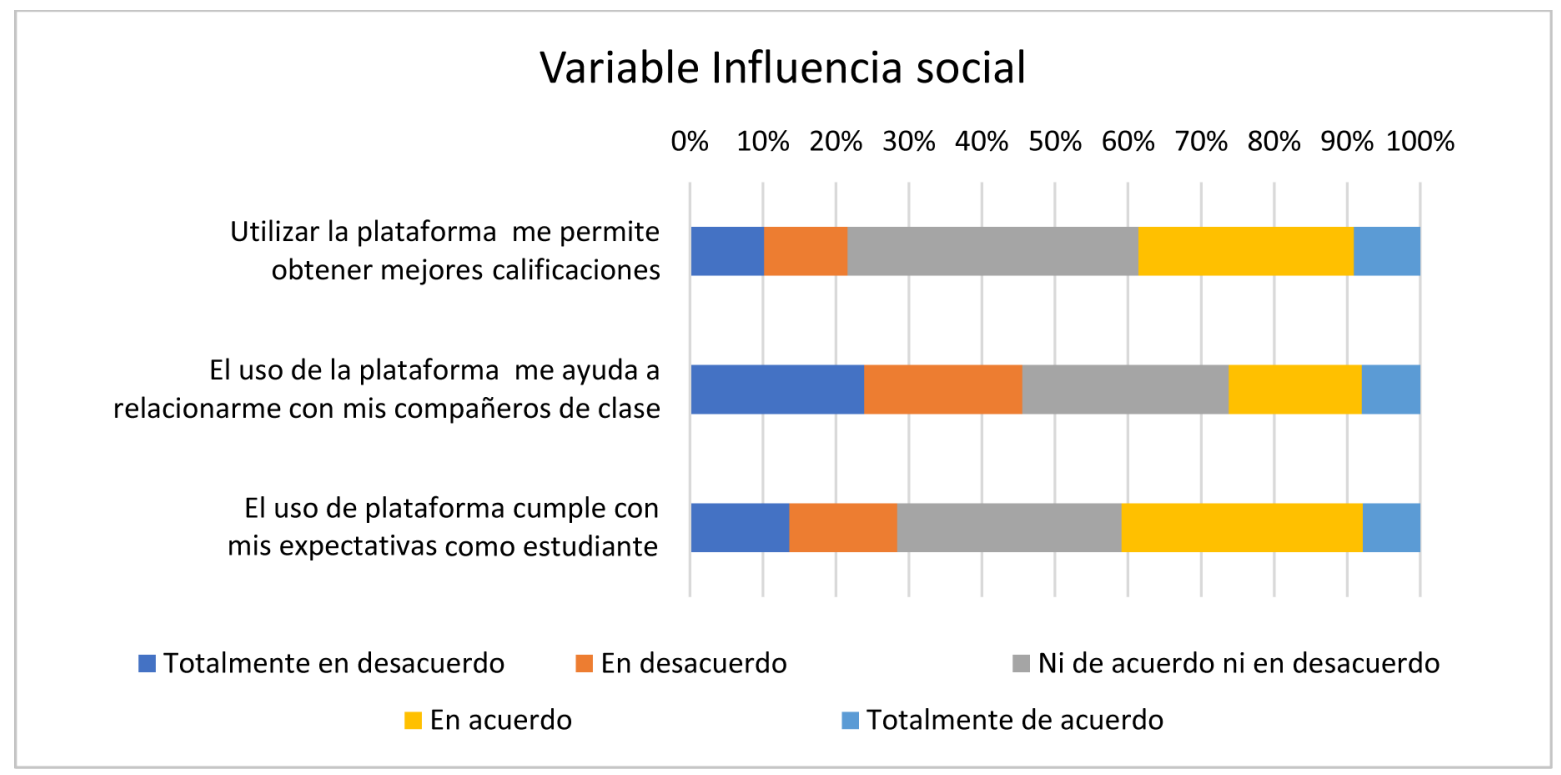

Figura 3. Resultados variable "Influencia social".

Fuente: Construcción propia.

resultados es que la media más alta con la desviación estándar más baja se encuentra en el ítem "Utilizar la plataforma me permite obtener mejores calificaciones"; un significado posible a este resultado es lo que indican Malhotra y Galleta (1999) respecto a que este criterio está relacionado con la identificación del individuo respecto a la tecnología utilizada, y sucede cuando el individuo acepta la influencia de un grupo de personas para mantener o mejorar la relación entre ellas, lo que en este caso no sucede.

Tabla 6. Media, desviación estándar y moda, variable "Influencia social".

\begin{tabular}{|c|c|c|c|}
\hline Ítem & $\overline{\mathrm{X}}$ & $\sigma$ & Moda \\
\hline 1. Utilizar la plataforma UNACH me permite obtener mejores calificaciones & 3.16 & 1.08 & 3 \\
\hline $\begin{array}{l}\text { 2. El uso de la plataforma UNACH me ayuda a relacionarme con mis } \\
\text { compañeros de clase }\end{array}$ & 2.65 & 1.25 & 3 \\
\hline $\begin{array}{l}\text { 3. El uso de plataforma UNACH cumple con mis expectativas } \\
\text { como estudiante }\end{array}$ & 3.07 & 1.16 & 4 \\
\hline
\end{tabular}

Fuente: Construcción propia.

\section{Influencia cultural}

En lo referente a los resultados de la variable "Influencia cultural", como se observa en la figura 4, el 11\% de los estudiantes manifestó estar totalmente de acuerdo en preferir trabajar solo que acompañado, mientras que el 21\% manifestó estar de acuerdo. Solo el $2 \%$ manifestó estar totalmente de acuerdo en que utilizar la plataforma los hace sentir cerca de sus compañeros aunque no los vean físicamente, seguido del 11\% 
que mencionó estar de acuerdo; mientras que para el ítem relativo a que los hombres tienen mejor rendimiento académico utilizando la plataforma en comparación con el rendimiento de las mujeres solo el 6\% estuvo totalmente de acuerdo, seguido del $2 \%$ que manifestó estar de acuerdo. Por otra parte, el 13\% manifestó estar totalmente de acuerdo y el 20\% de acuerdo en que el uso de la plataforma les permitirá tener mejor calidad de vida cuando se gradúen; mientras que el 22\% estuvo totalmente de acuerdo y el $25 \%$ de acuerdo en que el uso de la plataforma les brinda la certidumbre de saber qué actividades realizar. En cuanto a la percepción respecto a si el uso de la plataforma les permite hacer varias cosas al mismo tiempo, el 39\% estuvo totalmente de acuerdo, seguido del 32\% que estuvo de acuerdo. El último ítem de esta variable fue acerca de la percepción respecto a si consideran que la plataforma les ayuda ampliar su vocabulario, el 16\% estuvo totalmente de acuerdo y el 28\% de acuerdo.

\section{Variable Influencia cultural}

$\begin{array}{lllllllllll}0 \% & 10 \% & 20 \% & 30 \% & 40 \% & 50 \% & 60 \% & 70 \% & 80 \% & 90 \% & 100 \%\end{array}$

Prefiero trabajar solo que acompañado

Utilizar la plataforma me hace sentir cerca de mis compañeros aunque no los vea físicamente

Los hombres tienen mejor rendimiento académico utilizando la plataforma que las mujeres

El uso de la plataforma me permitirá tener mejor calidad de vida cuando me gradué

El uso de la plataforma me da la certidumbre de saber qué actividades realizar

El uso de plataforma me permite hacer varias cosas al mismo tiempo

La plataforma me ayuda a ampliar mi vocabulario

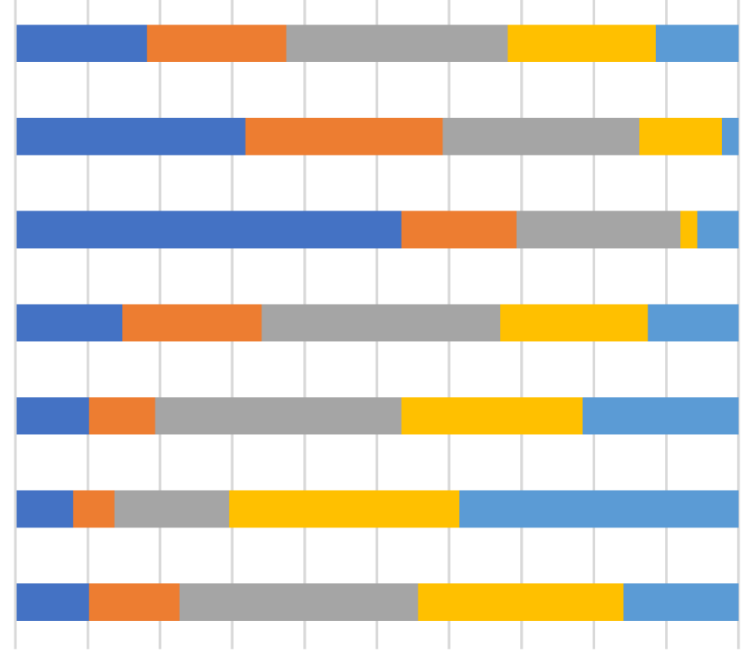

- Totalmente en desacuerdo

En desacuerdo

$\square$ Ni de acuerdo ni en desacuerdo

En acuerdo

- Totalmente de acuerdo

Figura 4. Resultados variable "Influencia cultural".

Fuente: Construcción propia.

Por otra parte, como se observa en la tabla 7, la media de la suma de todos los ítems de esta variable fue de 2.93, con una desviación estándar de 1.20 y una moda de 3 ; tomando en consideración estos resultados, es de llamar la atención lo relativo al ítem 6, en el cual se obtuvo una moda de 5 (totalmente de acuerdo), con una desviación estándar de 1.22 y una media de 3.88 (muy cercana a “de acuerdo"); mientras que en los ítems 2 y 3 se observa una moda de 1 (la más baja de todas las variables). 
Tabla 7. Media, desviación estándar y moda, variable "Influencia cultural".

\begin{tabular}{lccc}
\hline \multicolumn{1}{c}{ Ítem } & $\overline{\mathrm{X}}$ & $\sigma$ & Moda \\
\hline $\begin{array}{l}\text { 1. Prefiero trabajar solo que acompañado } \\
\begin{array}{l}\text { 2. Utilizar la plataforma UNACH me hace sentir cerca de mis compañeros } \\
\text { aunque no los vea físicamente }\end{array}\end{array}$ & 2.88 & 1.26 & 3 \\
\hline $\begin{array}{l}\text { 3. Los hombres tienen mejor rendimiento utilizando la plataforma } \\
\text { UNACH que las mujeres }\end{array}$ & 1.91 & 1.10 & 1 \\
\hline $\begin{array}{l}\text { 4. El uso de la plataforma UNACH me permitirá tener mejor calidad } \\
\text { de vida cuando me gradué }\end{array}$ & 2.97 & 1.23 & 1 \\
\hline $\begin{array}{l}\text { 5. El uso de la plataforma UNACH me da la certidumbre de saber } \\
\text { qué actividades realizar }\end{array}$ & 3.39 & 1.22 & 3 \\
\hline \begin{tabular}{l} 
6. La plataforma UNACH me permite hacer varias cosas al mismo tiempo \\
\hline
\end{tabular} & 3.88 & 1.22 & 5 \\
\hline
\end{tabular}

Fuente: Construcción propia.

\section{DisCUSIÓN Y CONCLUSIONES}

Los estudiantes que participaron en esta investigación son jóvenes considerados como "aprendices digitales", es decir, una nueva generación de estudiantes en la era digital, que poseen mucha afinidad y tendencia a utilizar la tecnología, sin embargo esto no significa que ello conlleve a garantizar que acepten el uso de todas las tecnologías disponibles. Las razones para no aceptar la tecnología pueden ser variadas, y van desde el hecho de no encontrar facilidad en el uso (asumiendo que poseen la tecnología en cuestión) hasta el de que no cuenten con los recursos necesarios para hacer uso de esta.

En esta ocasión, de acuerdo a los resultados encontrados queda de manifiesto que la utilización del LMS en la modalidad b-learning de la prueba piloto realizada aún tiene algunos desafíos para lograr la aceptación total de los estudiantes respecto al uso, así como de la percepción de su facilidad.

Estos desafíos pueden superarse si se enfocan esfuerzos en el diseño e implementación de estrategias que permitan a los estudiantes mejorar su desempeño escolar, así como otorgarles mayor capacitación respecto al uso del LMS en cuestión, debido a que aún no perciben que el LMS les ayude a ser mejores estudiantes, punto fundamental para obtener el mayor aprovechamiento posible de esta tecnología en favor del proceso enseñanza y aprendizaje, ya que de nada sirve que consideren que su uso es fácil si no le encuentran utilidad para mejorar su desempeño escolar.

Si bien es cierto que el b-learning brinda flexibilidad en tiempo y espacio, así como acceso a una gran variedad de recursos, también es cierto que implica mayor responsabilidad y autonomía por parte del estudiante, aunado a la evidente necesidad del apoyo, motivación y seguimiento continuo que brinden los profesores, tanto de manera presencial como a través del sistema de gestión del aprendizaje que se utilice; 
solo a través del acompañamiento oportuno y adecuado del docente se logrará justificar el uso de un LMS como complemento del proceso de enseñanza y aprendizaje.

Se considera que esta investigación cumplió con los objetivos planteados debido a que se determinó la utilidad y facilidad de uso percibida del LMS utilizado para la prueba piloto de la modalidad b-learning, mismos que fueron descritos en el apartado de resultados, de lo que se concluye que no existe percepción de utilidad hacia el uso, pero sí facilidad de uso percibida.

Por otra parte, respecto a los parámetros culturales y sociales que influyen en la percepción y facilidad de uso del LMS en cuestión, a continuación se presentan los las conclusiones más relevantes acerca de los hallazgos encontrados, vistos desde los criterios que se mostraron a través del diseño de los ítems:

Influencia social:

1. Cumplimiento: visto desde el comportamiento que adopta el estudiante para obtener un reconocimiento, que en este caso fue obtener mejores calificaciones. Al respecto se encontró que los estudiantes en su mayoría no perciben que el LMS les ayude a mejorar su desempeño escolar.

2. Identificación: que se interpretó a través de la opinión de los estudiantes respecto a si la plataforma les ayuda a relacionarse con sus compañeros de clase, lo cual significaría la influencia del grupo para mejorar la relación entre ellos; en este sentido el resultado indica que no existe influencia social, ya que en su mayoría los estudiantes opinaron estar en desacuerdo o totalmente en desacuerdo.

3. Internalización: visto cuando un individuo acepta la influencia, en este caso la plataforma, porque es congruente con lo que él/ella cree; en este sentido la mayor parte de los estudiantes manifestó que sí percibe la influencia social. Influencia cultural:

1. Individualismo: interpretado a través del ítem relativo al grado en que el estudiante prefiere trabajar solo que como miembro de un grupo; en este caso se concluye que sí existe influencia cultural, debido a que la mayoría de los estudiantes estuvo de acuerdo o totalmente de acuerdo.

2. Grado de distancia: visto como la cercanía o lejanía que el estudiante percibe respecto a sus compañeros de clase; al respecto se concluye que no se percibe influencia cultural debido a que la mayoría de los resultados estuvieron entre en desacuerdo y totalmente en desacuerdo.

3. Masculinidad/feminidad: interpretado a través del ítem que menciona que los estudiantes tienen mejor rendimiento académico utilizando la plataforma en comparación con las mujeres; al respecto se concluye que no existe percepción de influencia cultural, debido a que la mayoría de las respuestas fueron entre totalmente en desacuerdo y en desacuerdo. 
4. Calidad de vida laboral percibida: visto como la percepción del estudiante respecto a si considera que el uso de la plataforma contribuirá a que tenga mejor calidad de vida a futuro; al respecto el mayor porcentaje de respuesta se centra en la respuesta ni de acuerdo ni en desacuerdo, por lo que se concluye que no existe percepción cultural del LMS respecto a la mejora en la calidad de la vida futura de los estudiantes.

5. Evitar la incertidumbre: interpretada a través del ítem que indica si el uso de plataforma les da certidumbre respecto a qué actividades deben realizar; al respecto se concluye que sí existe influencia cultural, debido a que la mayoría de los estudiantes manifestó estar entre totalmente de acuerdo y de acuerdo.

6. Monocromía/policromía del tiempo: interpretada a través del ítem que indica que la plataforma les permite realizar varias actividades al mismo tiempo; en definitiva se concluye que sí existe esta influencia cultural, debido a que la mayoría de los estudiantes manifestó estar entre totalmente de acuerdo y de acuerdo.

7. Contexto de comunicación: el cual se aborda mediante el ítem que indica que el LMS ayuda a los estudiantes a ampliar su vocabulario; al respecto se concluye que sí hay que influencia cultural, debido a que el contexto cultural del vocabulario es capaz de modificar el comportamiento de las personas frente al uso de una tecnología determinada.

Yong, Rivas y Chaparro (2010) mencionan que algunos autores afirman que las personas que diseñan el desarrollo y la aplicación de TIC no toman en consideración las diferencias culturales de los usuarios, lo que podría tener algunas implicaciones, como por ejemplo que se utilicen mal o que su aplicación resulte fallida, por lo que para este caso en específico se recomienda realizar un análisis respecto al diseño de contenidos que se utilizó en la prueba piloto, con la intención de detectar las áreas de oportunidad específicas y plantear las estrategias necesarias que permitan su total aceptación y percepción de facilidad de uso.

\section{REFERENCIAS}

Adell, J., y Area, M. (2009). eLearning: Enseñary aprender en espacios virtuales. En J. De Pablos (coord): Tecnología Educativa. La formación del profesorado en la era de Internet (pp. 391-424). Málaga: Aljibe.

Ajzen, I. (1991). The theory of planned behavior. Organizational Behavior and Human Decision Processes, 50(2), 179-211. doi. org/10.1016/0749-5978(91)90020-T.

Ajzen, I., y Fishbein, M. (1980). Understanding attitudes and predicting social behavior. Englewood Cliffs, NJ: Prentice-Hall.

Belanger, F., y Jordan, D. (2000). Evaluation and implementation of distance learning: Technologies tools and techniques. Hersey: Idea Group.
Belloch, C. (2014). Diseño instruccional. Unidad de Tecnología Educativa. Universidad de Valencia. Recuperado de: https://www.uv.es/bellochc/pedagogia/EVA4.pdf.

Celina, O. H., y Campo-Arias, A. (2005). Aproximación al uso del coeficiente alfa de Cronbach. Revista Colombiana de Psiquiatría, 34(4), 572-580. Recuperado de: http:// www.scielo.org.co/pdf/rcp/v34n4/v34n4a09.pdf.

Davis, F. D. (1989). Perceived usefulness, perceived ease of use, and user acceptance of information technology. MIS Quarterly, 13(3), 319-340. doi:10.2307/249008. 
Gisbert Cervera, M., De Benito Crosetti, B., Pérez Garcias, A., y Salinas Ibáñez, J. (2018). Blended learning, más allá de la clase presencial. RIED. Revista Iberoamericana de Educación a Distancia, 21(1), 195-213. DOI: http://dx.doi.org/10.5944/ ried.21.1.18859.

Graham, C. R. (2006). Blended learning systems. Definition, current trends and future directions. En J. Curtis, Ch. Bonk y R. Graham (eds.), The handbook of blended learning: Global perspectives. Local designs. John Wiley \& Sons.

Graham, C. R. (2013). Emerging practice and research in blended learning. En M. G. Moore (ed.), Handbook of distance education (3a. ed., pp. 333-350). Nueva York: Routledge.

Herrera, M. A. (2006). Consideraciones para el diseño didáctico de ambientes virtuales de aprendizaje: una propuesta basada en las funciones cognitivas del aprendizaje. Revista Iberoamericana de Educación, 35(8). Recuperado de: https:/ / www.researchgate.net/publication/28108445_Consideraciones_para_el_diseno_didactico_de_ambientes_virtuales_de_aprendizaje_una_propuesta_basada_en_las_funciones_cognitivas_del_aprendizaje.

Leyton, S. D. A. (2013). Extensión al modelo de aceptación de tecnología TAM, para ser aplicado a sistemas colaborativos, en el contexto de pequeñas y medianas empresas [Tesis de Maestría]. Universidad de Chile. Recuperado de: http:/ / repositorio. uchile.cl/bitstream/handle/2250/115509/cf-leyton_ ds.pdf? sequence $=1$.

Martín, G. A., y Sánchez, G. M. (2014). Modelo predictivo de la intención de adopción de Blended learning en profesores universitarios. Universitas Psychological, 13(2), 601-614. DOI: https://doi.org/10.11144/Javeriana.UPSY13-2.

Malhotra, Y., y Galletta, D. F. (1999). Extending the technology acceptance model to account for social influence: Theoretical bases and empirical validation. Ponencia presentada en la 32nd Annual Hawaii International Conference on System Sciences (vol. 1, pp. 6-14), Maui, HI, EEUU.

McCoy, S., y Polak, P. (2003). Investigating the effects of national culture, infrastructure, and access costs on the use of information and 68 communication technologies in Mexico. 9th Americas Conference on Information Systems, Tampa, FL, EEUU. Salinas, J., Darder, A., y De Benito, B. (2015). Las TIC en la enseñanza superior: e-learning, b-learning y m-learning. En J. Cabero y J. Barroso (coords.), Nuevos retos en tecnología educativa (pp. 153-174). Madrid: Síntesis.

Salinas, I. J., De Benito, C. B., y Pérez, G. A. (2018). Blended learning, más allá de la clase presencial. Revista Iberoamericana de Educación a Distancia, 21(1). Recuperado de: http:/ / revistas.uned.es/index.php/ried/article/view/18859.

Vázquez, M. C., y Rodríguez, P. M. (2007). La deserción estudiantil en educación superior a distancia: perspectiva teórica y factores de incidencia. Revista Latinoamericana de Estudios Educativos, 37(3-4), 107-122.

Venkatesh, V., y Davis, F. D. (2000). A theoretical extension of the technology acceptance model: Four longitudinal field studies. Management Science, 46(2), 186-204. DOI: https:// doi.org/10.1287/mnsc.46.2.186.11926.

Venkatesh, V., y Bala, H. (2008). Technology acceptance model 3 and a research agenda on interventions. Decision Sciences, 39(2), 273-312. Recuperado de: https://www.academia. edu/9829534/Technology_Acceptance_Model_3_and_a_ Research_Agenda_on_Interventions.

Williams, P., Schrum, L., Sangrà, A., y Guàrdia, L. (2003). Fundamentos del diseño técnico-pedagógico en e-learning. Modelos de diseño instruccional. España: Universitat Oberta de Catalunya.

Yong, V. L., Rivas, T. L., y Chaparro, J. (2010). Modelo de aceptación tecnológica (TAM): un estudio de la influencia de la cultura nacional y del perfil del usuario en el uso de las TIC. Innovar. Revista de Ciencias Administrativas y Sociales, 20(36), 187-203. Recuperado de: https://www.redalyc.org/ articulo.oa?id=818/81819028014.

Zakour, A. B. (2004). Cultural differences and information technology acceptance. En 7th Annual Conference of the Southern Association for Information Systems (pp. 156-161). Recuperado de: https://pdfs.semanticscholar.org/c23f/56024b05bb6e 423ce3f77d15473136a3e0b8.pdf.

Cómo citar este artículo:

López Maldonado, N. E., y Valdés Godínes, J. C. (2020). Utilidad y facilidad de uso percibida: desafíos tecnológicos en una modalidad b-learning. IE Revista de Investigación Educativa de la REDIECH, 11, e938. doi: https://doi. org/10.33010/ie_rie_rediech.v11i0.938. 